\title{
Modeling Potential-Induced Degradation (PID) of Field-Exposed Crystalline Silicon Solar PV Modules: Focus on a Regeneration Term
}

\author{
Eleonora Annigoni ${ }^{1}$, Alessandro Virtuani ${ }^{1}$, Fanny Sculati-Meillaud ${ }^{1}$, Christophe Ballif ${ }^{1,2}$ \\ ${ }^{1}$ École Polytechnique Fédérale de Lausanne (EPFL), Institute of Microengineering (IMT), Photovoltaics \\ and Thin Film Electronics Laboratory, Neuchâtel, CH-2000, Switzerland \\ ${ }^{2}$ CSEM, PV-Center, Neuchâtel, CH-2000, Switzerland
}

\begin{abstract}
In this work, we further elaborate a model that was previously presented to simulate the effect of potential-induced degradation (PID) on the performance of field-exposed modules. We add to the predictive model a term aimed at describing the effect of regeneration during the night. We also investigate the impact of irradiance, temperature, bias, and load conditions on power regeneration. Laboratory PID-degraded mini-modules were subjected to indoor regeneration tests at different stress levels in order to model the contribution of each factor.

This allows us to implement into our PID predictive model, besides a degradation term, a second term describing the regeneration. Finally, we apply this model with outdoor climatic data to obtain a more realistic simulation of the power evolution over time of PID-affected PV modules in different climatic conditions.
\end{abstract}

\section{INTRODUCTION}

In this work, we aim at developing a comprehensive model to predict the performance of solar photovoltaic (PV) modules in operation as a consequence of the Potential-Induced Degradation (PID) effect (see e.g. [1] and [2]). We consider crystalline silicon (c-Si) PV modules with conventional p-type cells. In a recent contribution, we presented a model for PID power degradation (in [3]) based on laboratory accelerated ageing tests.

With the aim of simulating the actual performance of fieldexposed modules, we complement the model by including the contribution of a regeneration term. Power regeneration can occur at night or under illumination during hot and dry periods. In particular, we study the impact of irradiance, temperature, bias and load conditions on the power regeneration of PID degraded PV modules.

First we performed indoor tests on pre-degraded minimodules at different regenerating conditions. From the results of such tests we then develop a mathematical description of PID recovery (as can be found in other contributions e.g., [4] and [5]).

This allows us to express a more comprehensive equation modeling PID, with the inclusion of a regeneration term besides a degradation one [3]. This general expression can then be used, in combination with a set of input meteorological data for a specific location, to model the evolution of PID on PV modules in operation. As a first attempt, we apply the model using the parameters obtained for our test devices and by selecting three locations with different climatic conditions.

\section{PReVious Contribution: Degradation}

In [3] we presented a mathematical model for maximum power degradation, which we briefly recall. We subjected twocell mini-modules to accelerated aging in climatic chamber at different levels of the main stress factors (which, for degradation, are: temperature, $T$, relative humidity, $R H$, and voltage, $V$ ). The maximum power was measured at specified times during the tests. The final equation we obtained to describe the time-evolution of power degradation as function of the stress factors is the following:

$$
\frac{P_{\max }(t)}{P_{\max , 0}}=1-C_{V} \cdot V
$$

The quantity $P_{\max }(t)$ is the mini-module's power after $t$ hours of exposure to constant stress levels, and $P_{\max , 0}$ is the initial power. Such equation applies for modules exposed to negative voltage with respect to the ground, and $V$ is the absolute value of this potential difference. The coefficient $C_{V}$ contains the effects of $T, R H$, and time $t$ (see (2)), and is in agreement with an equation previously proposed by Hacke et al. in [5] ( $\kappa$ is the Boltzmann constant).

$$
C_{V}(T, R H, t):=A \cdot e^{-\frac{E a}{\kappa \cdot T}} \cdot R H^{B} \cdot t^{2}
$$

The values we obtained for the parameters by fitting our experimental results with equation 1 are: $A=6.0188$. $10^{-15} h^{-2}, B=4.43$ [-], and $E_{a}=0.86 \mathrm{eV}$.

\section{EXPERIMENTAL WORK: REGENERATION}

It is well known from field experience that the PID mechanism is reversible: modules performance can show some level of regeneration, for example when modules are not exposed to voltage (at nighttime) or under illumination during hot and dry periods [2]. The recovery process was subject of some recent studies. For instance, in [4] an equation for the regeneration in dark as function of the temperature was 
proposed. Authors of ref. [6] show that the recovery mechanism can be affected by irradiance too. However, to our knowledge, a clear relation describing the combined effect of temperature, irradiance, and voltage in the regeneration is still missing.

In this work we improve the model in [3] by adding a term that describes the recovery process.

We laminated at PV-Lab two-cell mini-modules (size $20 \mathrm{~cm}$ $\mathrm{x} 40 \mathrm{~cm}$ ), all samples featuring the same cell type (commercial mono crystalline silicon p-type cells). The modules are laminated in a glass/backsheet configuration, with soda-lime glass at the front, a polyethylene-based backsheet, and a commercial ethylene-vinyl acetate (EVA) as encapsulant material. All samples were prepared with the same processes, the same bill of material, and in a single batch. We simulated the presence of a metallic frame by means of an electrically conductive aluminum tape on the four sides. To degrade their performance, we subjected the mini-modules to PID tests in climatic chamber according to the procedure described in the proposed IEC Technical Specification for PID in c-Si modules (IEC TS 62804-1:2015-08). Mini-module's leads are shortcircuited and a negative voltage is applied between the cells and the grounded frame. The temperature was increased to $85^{\circ} \mathrm{C}$ instead of the specified $60^{\circ} \mathrm{C}$ in order to accelerate degradation. Electrical parameters of the mini-modules were determined by means of IV measurements at standard test conditions (STC: AM1.5G, $25^{\circ} \mathrm{C}, 1000 \mathrm{~W} / \mathrm{m}^{2}$ ) using a LED-halogen based sun simulator. The mini-modules were characterized initially and at the end of the degradation tests (96h).

After the degradation, the same mini-modules were subjected to regeneration tests. On the one hand, to investigate the regeneration occurring at night, tests were performed at different temperatures in dark and with no applied voltage. On the other hand, to reproduce the daily regeneration (that may occur during hot and dry periods), the samples were exposed to different irradiance levels. We also investigated for the first time the effect of (1) bias voltage during regeneration under irradiance, by applying a bias to some of the samples, and (2) of load conditions.

\section{A. Regeneration in the dark}

For the study of nighttime recovery, we subjected predegraded mini-modules to recovery in dark. The predegradation tests were performed for all samples in a climatic chamber at $85^{\circ} \mathrm{C} / 85 \% \mathrm{RH} /-1000 \mathrm{~V}$ for $96 \mathrm{~h}$. Regeneration tests were then performed in an oven, at different temperatures and under dry conditions. Samples were kept in open-circuit conditions.

By defining the relative recovery at time $t$ as:

$$
R R(t):=\frac{P_{\max }(t)-P_{\min }}{P_{i n i}-P_{\min }}
$$

where $P_{i n i}$ and $P_{\min }$ are the mini-module maximum power at, respectively, the beginning and the end of the degradation test, we obtained (see Fig. 1) the following relation for the relative recovery as function of the temperature, in agreement with [4]:

$$
R R(t)=1-\exp \left(-\left(\frac{t}{\tau(T)}\right)^{\beta}\right)
$$

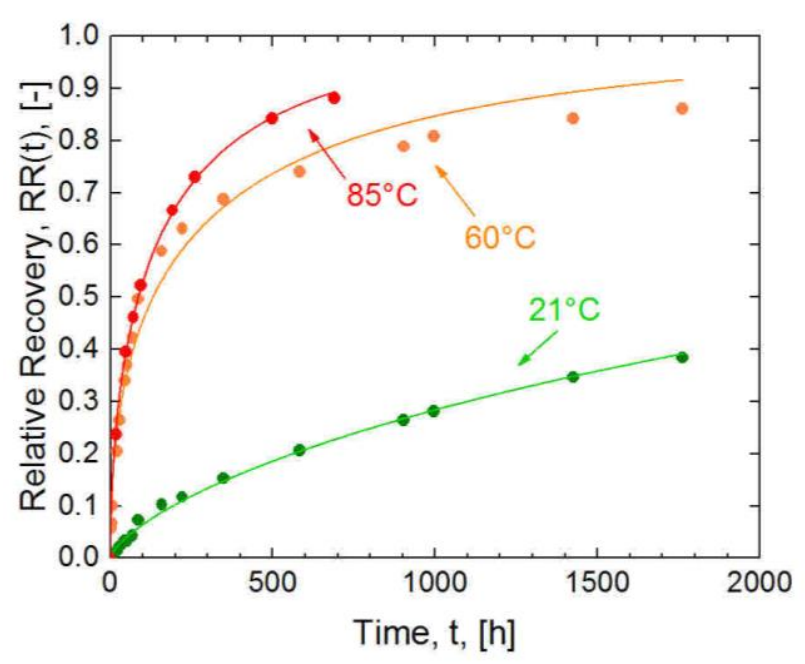

Fig. 1. Relative recovery over time for mini-modules during indoor testing in dark at different temperatures, with the fitting curves according to Eq. (4).

In Eq. (4), the parameter $\beta$ seems to be independent on temperature and has an average value of 0.55 [-]. The parameter $\tau[\mathrm{h}]$ follows an Arrhenius law with respect to temperature, with an activation energy of $0.52 \mathrm{eV}$.

The fact that higher temperatures induce a faster power recovery is consistent with the most accredited theory for the regeneration process, which attributes it to an out-diffusion of $\mathrm{Na}$ atoms from stacking faults in the pn junction [7]. As previously suggested in [4], this indicates that - under dry conditions - power regeneration might be more pronounced during daytime, with higher temperatures, than during nighttime, even in the presence of a negative applied bias to the modules. This is the main motivation behind this work.

\section{B. Regeneration during light exposure}

Here we aim at modeling the effect of irradiance on power recovery. As for regeneration in dark, all the samples were predegraded in climatic chamber at $85^{\circ} \mathrm{C} / 85 \% \mathrm{RH} /-1000 \mathrm{~V}$ for $96 \mathrm{~h}$. The recovery was then performed at different irradiance levels, in dry conditions (relative humidity around 15\%). A solar simulator system of class $\mathrm{C}$ was used that comprises a chamber with temperature controlled in a range of $\pm 5^{\circ} \mathrm{C}$. While the samples regenerating in the dark were kept at open-circuit (OC) conditions, here we wanted to reproduce the conditions at which fielded modules are kept during daylight. For this reason, 
we applied to some of the samples a negative voltage (simulating modules at the negative extreme of a string). In order to assess the impact on regeneration of irradiance only, other samples were left with no voltage applied (see Table I).

TABLE I

EXPERIMENTAL MATRIX OF TEST CONDITIONS TO INVESTIGATE THE REGENERATION MECHANISM

\begin{tabular}{|c|c|c|c|}
\hline \multirow{4}{*}{$\begin{array}{c}\mathbf{T} \\
{\left[{ }^{\circ} \mathbf{C}\right]}\end{array}$} & $\begin{array}{c}\text { Irradiance } \\
{\left[\mathbf{W} / \mathbf{m}^{2}\right]}\end{array}$ & $\begin{array}{c}\text { Load } \\
\text { conditions }\end{array}$ & $\begin{array}{c}\text { Voltage } \\
{[\mathbf{V}]}\end{array}$ \\
\hline \multirow{3}{*}{60} & \multirow{3}{*}{1000} & PID (SC) & -1000 \\
\cline { 3 - 4 } & & $\mathrm{SC}$ & $/$ \\
\cline { 3 - 4 } & & $\mathrm{OC}$ & $/$ \\
\cline { 3 - 4 } & \multirow{2}{*}{800} & $\mathrm{Mpp}$ & $/$ \\
\cline { 3 - 4 } & \multirow{2}{*}{640} & $\mathrm{PID}(\mathrm{SC})$ & -1000 \\
\cline { 3 - 4 } & & $\mathrm{MIDp}(\mathrm{SC})$ & -1000 \\
\cline { 3 - 4 } & & $\mathrm{Mpp}$ & $/$ \\
\hline
\end{tabular}

For a specific set of conditions $\left(1000 \mathrm{~W} / \mathrm{m} 2,60^{\circ} \mathrm{C},-1000\right.$ $\mathrm{V})$, we investigated the effect of load conditions as well, by exposing the modules, respectively, in short-circuit (SC), opencircuit $(\mathrm{OC})$, and, by applying a resistive load, maximum power point (Mpp).

Fig. 2 shows the relative power over time of the samples regenerating under $1000 \mathrm{~W} / \mathrm{m} 2$ at different load conditions. Two samples were tested per each condition.

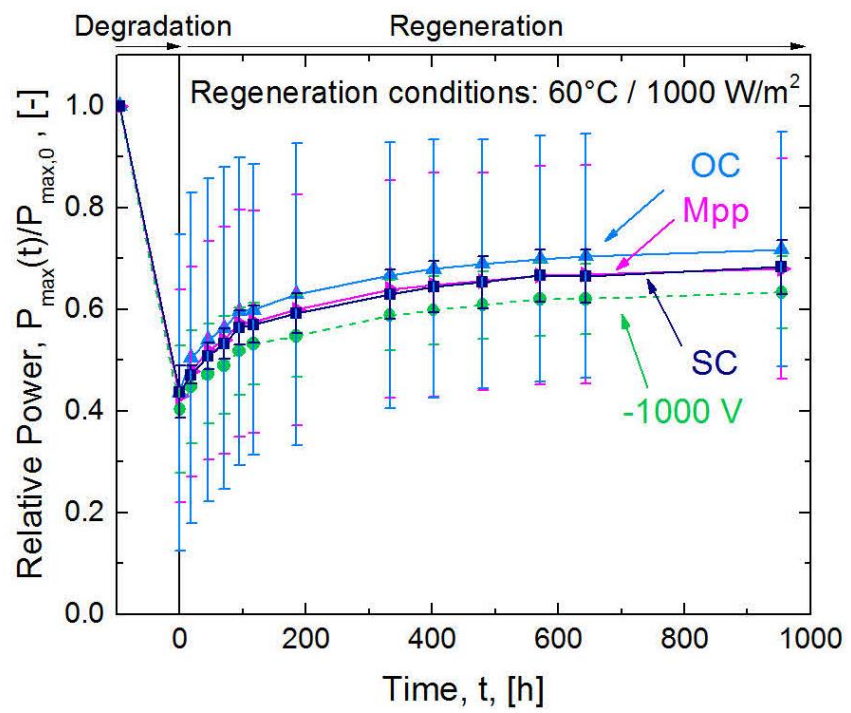

Fig. 2. Power evolution of mini-modules normalized by their initial power. The pre-degradation is performed at $85^{\circ} \mathrm{C} / 85 \% \mathrm{RH} /-1000 \mathrm{~V}$ for $96 \mathrm{~h}$. Regeneration starts at time $0 \mathrm{~h}$, with samples exposed to $60^{\circ} \mathrm{C}$, $1000 \mathrm{~W} / \mathrm{m}^{2}$ and at different load conditions. Relative humidity was low to enhance the regeneration (around 15\%). Per each load conditions, two samples were tested. Lines are a guide to the eye.
A slight difference can be seen in the regeneration speed and in the extent of maximum recovery. For instance, negativelybiased $(-1000 \mathrm{~V})$ mini-modules regenerate slower than the others, while samples in OC conditions show the strongest recovery, which we tend to ascribe to the slightly higher temperatures (a few degrees) of the devices held in OC. However, what needs to be highlighted is that, under these test conditions, even samples with a high negative voltage applied are regenerating. This means that daytime regeneration can occur in the field even for modules in strings exposed to high negative voltages towards ground, at least when humidity is low and/or in the absence of rain.

Results of regeneration under different irradiance levels are shown in Fig. 3. Dotted lines are for samples with $-1000 \mathrm{~V}$ applied, while straight lines represent samples in Mpp conditions. The effect of irradiance on the power regeneration seems not to be very pronounced, whereas a stronger impact is rather given by the load conditions.

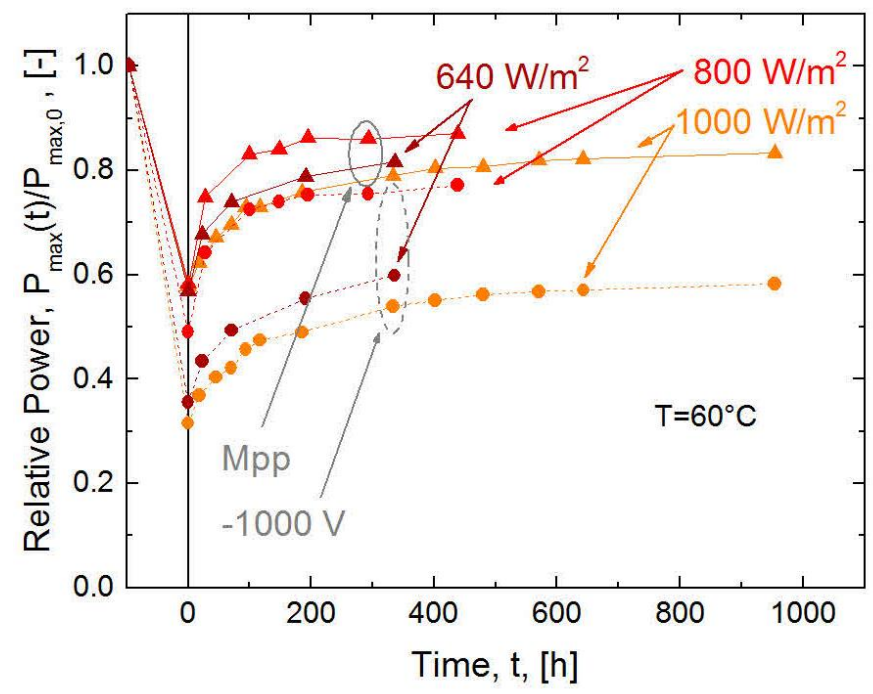

Fig. 3. Power evolution of mini-modules normalized to their initial power (before degradation). PID degradation is performed at $85^{\circ} \mathrm{C} /$ $85 \% \mathrm{RH} /-1000 \mathrm{~V}$ for $96 \mathrm{~h}$. Regeneration starts at time $0 \mathrm{~h}$, with samples exposed to $60^{\circ} \mathrm{C}$ and different irradiance levels. Relative humidity was low to enhance the regeneration (around 15\%). Lines are a guide to the eye. Dotted lines are for samples with a $-1000 \mathrm{~V}$ bias voltage applied, straight lines are for samples in Mpp conditions.

For the same samples, also the values of relative recovery (as defined in Eq. (3)) suggest that irradiance may have a secondary relevance in promoting the regeneration compared to load conditions (see Fig. 4). Here, however, the " $800 \mathrm{~W} / \mathrm{m}^{2} /-1000$ V" data-set contradicts partly these observations. One explanation for this may lie in possible temperature variations from the set value of $60^{\circ} \mathrm{C}$ in the different tests: samples regenerating faster might actually be exposed to slightly higher temperatures than the others. We then speculate that irradiance might have only a secondary relevance in promoting regeneration, whereas temperature plays a larger role. Further tests are ongoing to definitely assess the contribution of irradiance on power recovery. 
In addition, even if - in order to avoid contradictory results particular care has been dedicated in sample processing (same bill of material, processes, and processing time), the samples show different level of degradation (i.e. not all samples degrade by the same amount), as can be seen in Fig 3. This variability then translates into different levels of recovery or of relative recovery (see Fig. 4), which may have a considerable impact on our findings/conclusions. Further, it is widely observed (and reported in the literature) that the samples undergoing a more severe degradation tend to recover less.

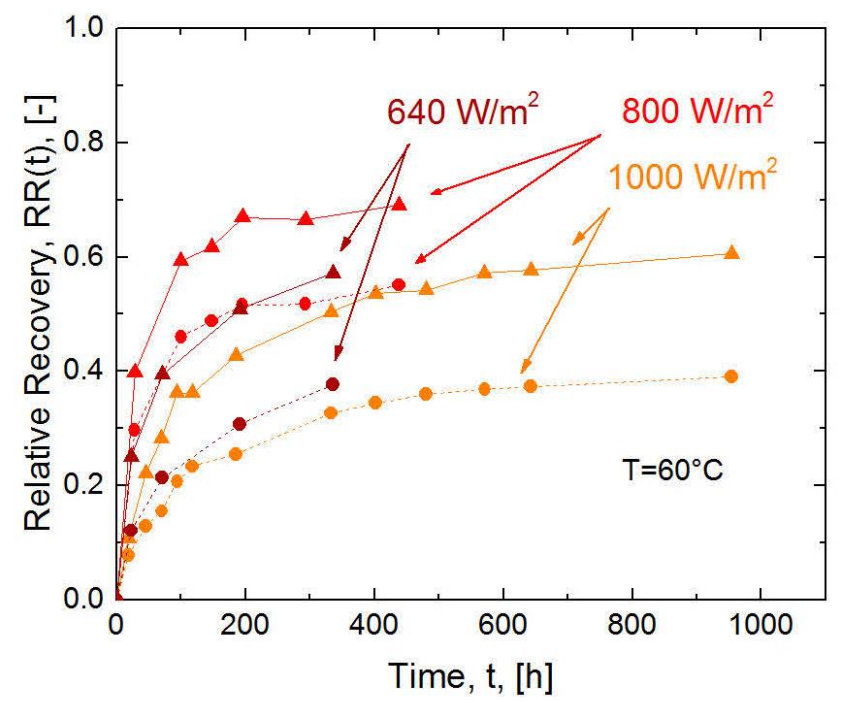

Fig. 4. Relative recovery (defined in Eq. (3)) referred to the same samples as in Fig. 3.

In order to complete the model for daytime power recovery, on-going work (not presented in this paper) is investigating, for a fixed irradiance level (i.e. $1000 \mathrm{~W} / \mathrm{m}^{2}$ ), the effects that (1) temperature and (2) bias voltage have on the regeneration. The latter to assess the distribution of the recovery process as a function of the position of the module in a string.

\section{SIMULATING OUTDOOR PID}

The final goal of this work is to model the evolution of the PID phenomenon and the impact on module performance as a function of different climatic conditions. To do this we applied the equations determined by means of indoor accelerated tests (Eq. (1) and (4)), with Typical Meteorological Year data as input parameters for the simulations.

Applying these equations, determined at constant stress laboratory conditions, to outdoor weather data is not straightforward. Indeed, indoors module's power undergoes a continuous degradation described by (1) and by a fixed initial value $P_{\max , 0}$ (and analogously for regeneration), whereas outdoors $P_{\max }(t)$ fluctuates continuously depending on the time-varying temperature, irradiance, and other environmental parameters. Our approach is described in [3]; in particular we employ the concept of equivalent time to make the implementation with varying stress conditions consistent.

Here we complete the model by adding the contribution of the regeneration process.

In Fig. 5, by using the indoor-determined parameters of our test devices, we simulate the power of a 2-cell mini-module exposed to $-1000 \mathrm{~V}$ in three different climates. We assume, as a first approximation, that degradation occurs during daytime, according to Eq. (1), and thermally-driven recovery during nighttime, Eq. (4). Degradation is supposed to occur for any level of relative humidity (according to (4)), differently from other works, such as [8], where it is supposed to apply only when relative humidity is above a certain threshold level. Simulation results show a clear impact of relative humidity on the different degradation levels reached in the three climates. One can observe that the effect of seasonality is also evident, e.g. in Miami the trade-off between degradation and regeneration is well in correspondence with the rain and the dry season respectively of this location. A similar seasonal trend was observed in [2] for modules installed in PID conditions in Florida.

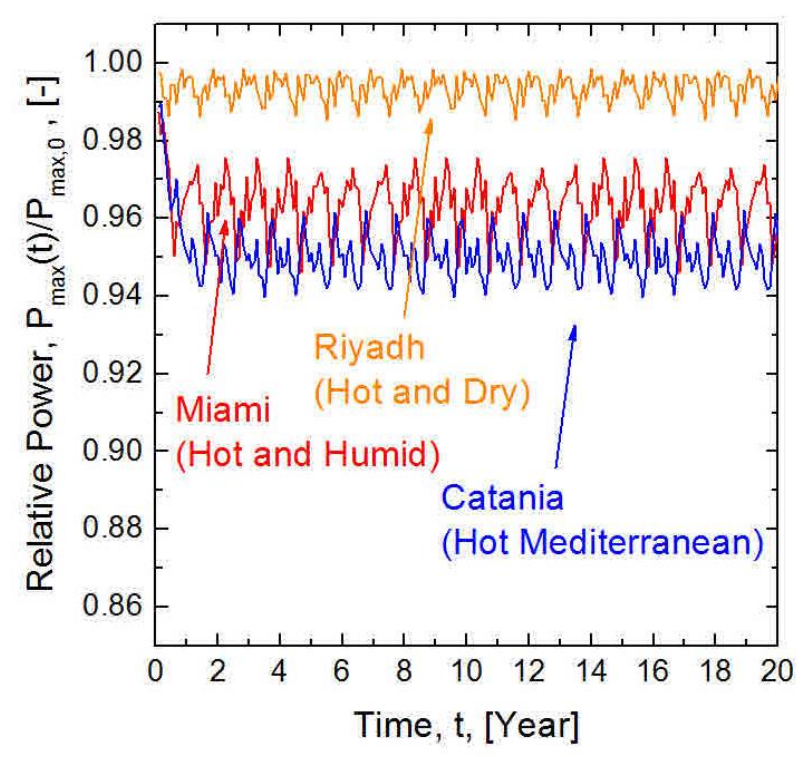

Fig. 5. Simulated time-evolution of a 2-cell mini-module's power as consequence of daytime PID and nighttime regeneration in three different climates.

The planned experimental work described in the previous section should clarify more the dependence of power regeneration on irradiance. We will then integrate the term for daytime regeneration in the simulations.

As the model presented in this work is obtained for 2-cell mini-modules, it also needs to be adapted to full-size modules taking into account the different voltage that affects each cell. This extension of the model will be based on previous works, such as [6] and [8]. 


\section{SUMMARY}

We present an improved model for PID prediction, based on a study of the regeneration mechanism. For the first time, the relationship between power recovery and irradiance, temperature, voltage, and load conditions was investigated, by means of regeneration tests performed at different stress levels.

In dark conditions (replicating nighttime recovery), the power regeneration is promoted by higher temperatures according to an Arrhenius behavior, in agreement with what found in [4].

Under illumination, what needs to be highlighted is that, under these specific test conditions (illumination, $60^{\circ} \mathrm{C}$, low humidity), regeneration clearly takes place as well for the samples exposed to a considerable high negative bias-voltage ($1000 \mathrm{~V})$. The results obtained so far suggest that different irradiance levels do not have a strong effect on the power regeneration, whereas a clear impact is given by the load conditions. However, in spite of the accuracy with which we manufactured the samples in order to guarantee the repeatability of the results (all samples were laminated on the same day and with the same roll of encapsulant and of backsheet), we still obtain a quite high variability in the results. One possible reason could be that the temperature in the different tests had some variations from the set value of $60^{\circ} \mathrm{C}$, so that samples exposed to a higher temperature could regenerate faster than the others. In view of these preliminary results we speculate that irradiance may play a secondary role in the recovery mechanism, whereas temperature is a more relevant factor.

The results of regeneration tests performed so far allow us modeling the term for nighttime regeneration, which we included in our previous predictive model for degradation, [3]. Finally, we apply this PID model with outdoor climatic data to obtain a realistic simulation of modules performance in different climatic conditions, see Fig. 5. A trade-off between power degradation and regeneration is reproduced, and it matches the yearly alternation of the humid and the dry season. Such seasonal effect in the case of Miami climate is well in correspondence with that observed in [2] for modules exposed outdoors in Florida.

\section{ACKNOWLEDGMENTS}

We gratefully acknowledge the financial support of EOS Holding and of the Swiss National Science Foundation (SNSF) through the National Research Program (NRP) "Active Interfaces" (NRP 70). We are grateful to Xavier Niquille for support in the experimental work and to the all PV-lab team.

\section{REFERENCES}

[1] S. Pingel, O. Frank, M. Winkler, S. Daryan, T. Geipel and H. B. J. Hoehne, "Potential Induced Degradation of Solar Cells and Panels," 2010.

[2] P. Hacke, R. Smith, K. Terwilliger, G. Perrin, B. Sekulic and S. Kurtz, "Development of an IEC test for crystalline silicon modules to qualify their resistance to system voltage stress," Progress in Photovoltaics: Research and Applications, vol. 22, pp. 775-783, 2014.

[3] E. Annigoni, M. Jankovec, F. Galliano, H. Li, L. PerretAebi, M. Topic, F. Sculati-Meillaud, A. Virtuani and C. Ballif, "Modeling potential-induced degradation (PID) in crystalline silicon solar cells: from accelerated-aging laboratory testing to outdoor prediction," in 32nd EU PVSEC, Munich, 2016.

[4] P. Lechner, S. Hummel and J. Schnepf, "Evaluation of Recovery Methods after Potential Induced Degradation of PV Modules," in 31st European Photovoltaic Solar Energy Conference and Exhibition, Hamburg, 2015.

[5] P. Hacke, S. Spataru, K. Terwilliger, G. Perrin, S. Glick, S. Kurtz and J. Wohlgemuth, "Accelerated Testing and Modeling of Potential-Induced Degradation as a Function of Temperature and Relative Humidity," IEEE Journal of Photovoltaics, vol. 5, no. 6, pp. 1549-1553, 2015.

[6] S. Koch, J. Berghold, C. Hinz, S. Krauter and P. Grunow, "Improvement of a Prediction Model for Potential Induced Degradation by Better Understanding the Regeneration Mechanism," in 31 st European Photovoltaic Solar Energy Conference and Exhibition, Hamburg, 2015.

[7] D. Lausch, V. Naumann, A. Graff, A. Hähnel, O. Breitenstein, C. Hagendorf and J. Bagdahn, "Sodium outdiffusion from stacking faults as root cause for the recovery process of potential-induced degradation (PID)," Energy Procedia, vol. 55, pp. 486-493, 2014.

[8] J. Hattendorf, W.-M. Gnehr, R. Loew, T. Roth , D. Koshnicharov and M. Zentgraf, "Potential-induced degradation and temperature-driven regeneration: a realistic simulation," Paris, 2013. 